\title{
La inflación como factor distorsionante de la información financiera*
}

\author{
De La Hoz, Betty** \\ Uzcátegui, Sigilfredo*** \\ Borges, Jesús**** \\ Velazco, Angel ${ }^{* * * * *}$
}

\section{Resumen}

El propósito de este trabajo es analizar la inflación como factor distorsionante de la información financiera. La investigación se centra en las técnicas de medición de la inflación y el inicio del proceso de actualización o reexpresión de los datos históricos arrojados por los estados financieros, así como también, su incidencia bajo un escenario en Venezuela para el año 2006. Se efectuó una revisión documental bibliográfica basada en los fundamentos teóricos y estadísticos de: Fernández (2002), Catacora (2000), Yanes (2006), Banco Central de Venezuela (2006), entre otros, sobre la inflación, su medición, cálculos e incidencia. Los resultados arrojaron cómo la unidad monetaria en Venezuela, ha perdido su capacidad adquisitiva producto del fenómeno inflacionario, el cual se refleja en la información financiera de las empresas. Se concluye que los estados financieros de una empresa, constituyen el medio principal para comunicar información acerca de su situación financiera y re-

Recibido: 05-12-07. Aceptado: 06-02-08

* Este artículo es parte del proyecto de investigación: Variación del resultado histórico en el mejoramiento de la información financiera actualizada. Caso VENMACA adscrito al instituto de investigaciones de la Facultad de Ciencias Económicas y Sociales (FCES) de la Universidad del Zulia (LUZ). Financiado por el Consejo de Desarrollo Científico y Humanístico (CONDES).

** Contador Público. Magíster Scientiarum en Gerencia de Empresas, mención: Gerencia Financiera. Docente de la Universidad Rafael Urdaneta (URU), Asistente de Investigación del Instituto de Investigaciones de la facultad de Ciencias Económicas Sociales de la Universidad del Zulia. E-mail: betty.hoz@hotmail.com

*** Contador Público. Magíster en Gerencia de Empresas, mención: Gerencia Financiera. Docente e investigador del Instituto de Investigaciones de la Facultad de Ciencias Económicas y Sociales de la Universidad del Zulia. E-mail: suzcategui@gmail.com

**** Sociólogo. Magíster Scientiarum en Economía y Planificación del Desarrollo, mención: Planificación del Desarrollo. Docente jubilado e investigador del Instituto de Investigaciones de la facultad de Ciencias Económicas Sociales de la Universidad del Zulia.

E-mail: danielborges53@hotmail.com

***** Contador Público. Especialista en Gerencia Tributaria. Docente e investigador del Instituto de Investigaciones de la facultad de Ciencias Económicas Sociales de la Universidad del Zulia. E-mail: profangelvelazco@hotmail.com 
sultados de operación para la toma de decisiones, sin embargo, en economías inflacionarias los datos contables basados en costos históricos, presentan información distorsionada poco veraz, actual, relevante y precisa; por lo cual se recomienda actualizarla por efectos de la inflación.

Palabras clave: Información financiera, Capacidad adquisitiva, Inflación, Índice de precios al consumidor, partidas monetarias y no monetarias.

\title{
Inflation as a Distorting Factor in Financial Information
}

\begin{abstract}
The purpose of this study is to analyze inflation as a factor that distorts financial information. The research centers on inflation-measuring techniques and the beginning of the process for updating or re-expressing historical data produced by the financial statements, as well as its incidence on the Venezuelan scene for the year 2006. A documentary, bibliographical review was made based on the theoretical and statistical fundamentals of: Fernández (2002), Catacora (2000), Yanes (2006), and the Central Bank of Venezuela (2006), among others, regarding inflation, its measurement, calculation and incidence. Results indicated how the monetary unit in Venezuela has lost its purchasing power as a product of the inflationary phenomenon, which is reflected in the financial information of the companies. Conclusions are that the financial statements of a company constitute the principal means for communicating information about its financial situation and operational results for making decisions; nevertheless, in inflationary economies, accounting date based on historic costs presents distorted information that is hardly truthful, up-to-date, relevant or precise. Therefore, it is recommended to update financial statements for inflationary effects.
\end{abstract}

Key words: Financial information, purchasing power, inflation, consumer price index, monetary and non-monetary items.

\section{Introducción}

Los estados financieros, cuyo objetivo es suministrar a los usuarios información acerca de la situación financiera (Balance General), el resultado de la gestión económica (Estado de Resultados) y los cambios en la posición financiera o en la inversión por parte de los dueños (Estado de Flujos del efectivo o Cambios en las Cuentas del Patrimonio), son de gran utilidad para el proceso de toma de decisiones de las empresas. Dicha información debe estar preparada no solo para satisfacer las exigencias de la gerencia de la empresa, propietarios, trabajadores, acreedores, potenciales inversionistas, entre otros, en la toma de decisiones de índole económica, sino también la de una gran variedad de usuarios.

Sin embargo, el proceso inflacionario que por años se ha desarrollado en Venezuela, distorsiona la información presentada en los estados financieros de las empresas y su interpretación, por ser éstos preparados sobre la base de costos históricos; situación negativa para las empresas puesto que la información financiera constituye el fundamento de la toma de decisiones.

Con la inflación no solamente se ha perdido el poder adquisitivo del bolívar, sino la unidad de medición que mantuvo hasta la década de los ochenta, periodo 
La inflación como factor distorsionante de la información financiera

De La Hoz, Betty; Uzcátegui, Sigilfredo; Borges, Jesús y Velazco, Angel

en el que se le reconocía como una de las monedas más fuertes, no solo del continente americano, sino del mundo; y es precisamente allí donde radica el problema, al no reconocérsele como un fenómeno netamente monetario y financiero, el cual debe cuantificarse y registrarse.

La presencia del fenómeno económico de la inflación en Venezuela, obliga a separar en los resultados mostrados como ingrediente nuevo que exige la Contabilidad Reexpresada, los que provienen de la comercialización de los productos o servicios que presta la empresa, de aquellos resultados originados por el manejo o tratamiento de la gerencia sobre las partidas monetarias, para que de esta manera cualquier usuario pueda distinguir adecuadamente el efecto de la inflación en la información financiera.

Puesto que la inflación es un fenómeno económico que ha afectado a Venezuela por más de veinte años, desaparecerla o al menos neutralizarla, es uno de los objetivos básicos de los analistas económicos y preocupación constante de todos los Gobiernos venezolanos.

En América la inflación es un fenómeno persistente, por ejemplo, según Vera (1996), en el siglo pasado numerosos han sido los casos en los cuales se han registrado procesos inflacionarios importantes, como los de Bolivia (19841985) y Brasil (1989); y los índices inflacionarios de los últimos años en países latinomericanos han ido en aumento. Tal es el caso de Venezuela, país con el mayor índice inflacionario de Latinoamérica al presentar para el año 2005 la mayor inflación de la región, con un $14.4 \%$ y mantener el liderazgo también para el 2006 , con $17 \%$; le sigue Argentina con $9.8 \%$; mientras que con la inflación más baja de América Latina se presenta Perú con el 1.1\% (Centro de Estudios Latinoamericanos: 2007). Para el cierre del 2007, los especialistas pronostican el mismo escenario de liderazgo cerrando Venezuela con un $20 \%$.

Por lo antes señalado, no existen indicios que permitan considerar que la inflación desaparezca en el país, por lo menos a corto plazo. Aunque sus efectos y magnitudes se reduzcan, siempre será un aspecto distorsionante de la información financiera que suministran los Estados Financieros históricos, cuando son utilizados para la toma de decisiones gerenciales.

Se abordará la investigación desde una óptica conceptual basada en la definición de términos básicos asociados con la inflación, su medición, cálculo y la comparabilidad de las cifras que componen los estados financieros, para luego explicar los diferentes tipos de posiciones monetarias que se pueden obtener en un periodo determinado así como los resultados monetarios del ejercicio y su respectiva interpretación. Se cierra la investigación exponiendo la interrelación que puede existir entre la ecuación fundamental de contabilidad y la actualización de la información financiera por efectos de la inflación.

\section{Definición de Inflación}

Fernández (2002) define la Inflación como un fenómeno de alza pronunciada, continua, generalizada y desordenada de los precios de bienes y servicios que trae como consecuencia una pérdida sensible del poder adquisitivo de la unidad monetaria del país. Destaca que so- 
lamente el cambio en los niveles generales de precios debe ser tomado como inflación, y no el cambio producido a nivel específico de un bien o servicio. Según Fernández (2002), la inflación puede ser considerada como una enfermedad de la economía cuya causa principal es la existencia de mucho circulante en poder del público y poca producción de bienes y servicios. Esta situación hace que cumpliendo con la teoría de la oferta la demanda se produzca un alza de precios al existir mayor demanda que oferta.

Por su parte, Catacora (2000), explica que la inflación es el incremento porcentual de los precios de una cesta de bienes y servicios representativa de una economía, entre dos períodos determinados. Es un fenómeno económico que genera diversas consecuencias, como la pérdida del poder adquisitivo de la moneda o del signo monetario representativo de una economía. En relación con esta definición, Dornbusch, Fischer y Startz (2002), señalan que la inflación es la tasa de variación de los precios durante un período determinado.

Por otra parte, Redondo (1993) dice que la inflación es el deterioro del poder adquisitivo de un signo monetario empleado como patrón de medida en el intercambio de bienes o servicios, incrementando el valor monetario de los mismos con lo cual disminuye el poder de compra de esa moneda. Agrega que el fenómeno de la inflación no es nuevo, más bien la humanidad lo viene soportando desde hace mucho tiempo, pero en especial desde que se inventó la moneda como signo de cambio.

En este mismo orden de ideas, Vera (1996), indica que la inflación genera costos para la sociedad, y que en sus fases iniciales crea cambios bruscos en los precios relativos, deteriora los saldos reales, afecta la liquidez de las unidades económicas, profundiza los problemas distributivos, beneficia a los deudores monetarios y perjudica a los acreedores. Agrega además, que la inflación alta es una fuente de desequilibrio de las finanzas públicas, crea desconfianza en la moneda doméstica, desalienta la inversión productiva y reduce el ritmo de acumulación de la economía.

De acuerdo a lo planteado en los párrafos anteriores, se puede notar que la inflación afecta directamente a todo aquello que tiene que ver con dinero o con partidas circulantes, por lo que en un país con inflación como Venezuela, se dificulta la comprensión y utilización de los informes financieros emitidos por las empresas, debido a la distorsión que estos sufren. De allí que la Declaración de Principios de Contabilidad Número 10 (DPC-10), indique que los estados financieros expresados en cifras históricas y en términos de unidades monetarias que se consideren constantes pueden tener validez en una economía sin inflación, pero evidentemente cuando los precios aumentan en forma general, los valores se distorsionan y los estados financieros no proporcionan información adecuada para:

- La gestión empresarial.

- La evaluación de proyectos.

- La elaboración de presupuestos.

- Las oportunidades de inversión.

- El pago de dividendos.

- La evaluación de la tasa real de rentabilidad de las inversiones.

- El cálculo del valor económico agregado. 
La inflación como factor distorsionante de la información financiera

De La Hoz, Betty; Uzcátegui, Sigilfredo; Borges, Jesús y Velazco, Angel

- La determinación efectiva de la tasa de impuestos.

- La comparación de información con períodos distintos.

La DPC-10 en su párrafo 34 dice que Venezuela sigue presentando altos niveles de inflación que distorsionan la información financiera, que originarían la descapitalización de las empresas por efecto de impuestos y dividendos, y que harían inútil la información financiera presentada en cifras nominales.

En este sentido, según Catacora (2000), en economías inflacionarias la contabilidad basada en costos históricos no permite que los estados financieros cumplan con sus características básicas. El párrafo 18 de la Declaración de Principios de Contabilidad número 0 (DPC-0), establece lo siguiente:

"Los estados financieros forman parte del proceso de presentar información financiera y constituyen el medio principal para comunicarla a las partes que se encuentran fuera de la entidad. Estos estados normalmente incluyen un balance general, un estado de resultados o de ganancias y pérdidas, un estado de movimiento de las cuentas de patrimonio, un estado de flujo de efectivo las notas a los estados financieros. El objetivo de los estados financieros es suministrar información acerca de la situación financiera de una entidad económica a una fecha determinada los resultados de sus operaciones los movimientos de su efectivo por los períodos en esa fecha entonces terminados".

Este párrafo tomado de la DPC-0, establece claramente que los estados financieros constituyen un medio impor- tantísimo para comunicar información a usuarios tanto internos como externos, por lo que es necesario que los datos reflejados en dichos estados financieros reflejen su verdadero valor actual. Para toda empresa que realice sus actividades en países con economías inflacionarias, es fundamental identificar todas sus partidas monetarias de un período específico y determinar si la inflación ha tenido en sus resultados de operación un efecto positivo o negativo, después de aplicar una metodología de actualización o ajuste de sus cifras aplicable en el país donde se realicen las operaciones.

Un aumento generalizado de precios o, lo que es lo mismo, la pérdida del poder adquisitivo de la moneda, significa que la unidad monetaria, es decir, el bolívar, pierde estabilidad, no solo en cuanto a su capacidad adquisitiva para determinados bienes y servicios que las empresas transforman, producen, comercializan y registran, sino respecto a todo lo que el país produce o importa. En otras palabras, la inflación anuló el concepto tradicional que tenia el Bolívar de unidad monetaria estable.

Ahora bien, el problema no es solo ese, sino que cuando por definición se refiere al aumento generalizado, se debe entender que el aumento en los precios de los bienes y servicios es relativo, o sea, el aumento es desigual entre los distintos bienes y servicios que intervienen en la economía, por lo tanto, el efecto o impacto que produce es muy diferente de persona a persona, de empresa a empresa, de sector a sector, y es por ello que genera una transferencia de riqueza y en algunos de los casos la 
pérdida de la riqueza al no tratarse debidamente (Vives, 2003).

La inflación al producir cambios en los precios, distorsiona la comparación de cifras de un periodo a otro, por lo que el valor de los activos cambia para las empresas con el transcurrir del tiempo, lo que consecuentemente hace que los estados financieros, presenten una imagen distorsionada o, en casos mas graves, una situación financiera totalmente alterada de la entidad, por lo que se hace necesario que la contabilidad recurra a una serie de normas y medidas para corregir esta situación. En el caso de Venezuela, las técnicas y pasos a seguir para la reexpresión de estados financieros por efectos de la inflación, se describen en la DPC-10 (Declaración de Principios de Contabilidad \# 10) promulgada por la Federación de Colegios de Contadores Públicos de Venezuela, el 23/08/91 y reemplazada en su totalidad por la normativa revisada e integrada 06/12/2000.

\section{Comparabilidad de las cifras de los estados financieros}

La comparabilidad contenida actualmente dentro del marco conceptual de las Normas Internacionales de Contabilidad (NIC), expresa que los usuarios deben ser capaces de comparar los estados financieros de una empresa a través del tiempo, a fin de identificar tendencias en la posición financiera y en sus resultados. Asimismo, los usuarios deben ser capaces de comparar los estados financieros de diferentes empresas con el objeto de evaluar su posición financiera, resultados y cambios en la posición financiera.
Este atributo, entre otros que hace útil la información suministrada por los estados financieros, no se obtiene en una economía inflacionaria como la de Venezuela, al pretender compararlos utilizando el esquema de los valores históricos. Por lo tanto, para poder explicar con ejemplos prácticos la no procedencia de esta comparación, es necesario aclarar algunos términos básicos:

- Medición de la inflación:

Es una técnica utilizada para conocer los niveles de inflación alcanzados por países con inflación. En Venezuela, por ejemplo, la inflación se mide a través de la variación porcentual del Índice de Precio al Consumidor (IPC) durante un periodo determinado, bien sea un mes, trimestre, semestre o año. (Banco Central de Venezuela (BCV): 2000).

- Índice de Precio al Consumidor (IPC):

Según el BCV (2006), en el boletín económico informativo de los meses de Marzo y Abril del año 2006, el IPC es un indicador estadístico que mide el cambio promedio registrado en los precios a nivel de consumidor, para una canasta de bienes y servicios representativos del consumo familiar, en un período determinado.

- Año Base:

Es un año de referencia adoptado para el cálculo del IPC, cuyo nivel inicial es 100. En Venezuela, a la fecha, el año base es 1997 (BCV, 2000).

- Tasa de Inflación:

Es un cociente que resulta de la comparación entre dos índices de precios. Para calcular, por ejemplo, la tasa de inflación registrada en el año 2006, para el área metropolitana de Caracas, se toman como referencia los niveles del 
La inflación como factor distorsionante de la información financiera

De La Hoz, Betty; Uzcátegui, Sigilfredo; Borges, Jesús y Velazco, Angel

IPC de Diciembre 2006 y Diciembre 2005, aplicándose la siguiente fórmula:

TASA INFLACIÓN $=\left(\frac{\text { IPCdic }-2006}{I P C d i c-2005}-1\right) * 100$

Sustituyendo los valores, se tiene:

$$
\begin{aligned}
\text { TASA INFLACIÓN } & =\left(\frac{614.83179}{525.64893}-1\right) * 100 \\
& =(1.16966-1)^{*} 100 \\
& =16.97 \%
\end{aligned}
$$

- Tasa de Inflación Promedio:

Es el cociente que resulta de comparar dos índices de precios promedios, el cual se acostumbra publicar en la prensa escrita a principios de cada año, utilizando para estos casos los valores promedios. Por ejemplo, para calcular la tasa de inflación promedio para el 2006, se toma como referencia el IPC promedio del año 2006 y el IPC promedio del año 2005. La fórmula para su cálculo es la siguiente:

\section{TASA INFLACIÓN PROMEDIO =}

$\left(\frac{I P C \operatorname{Pr} \text { om }-2006}{I P C \operatorname{Pr} \text { om }-2005}-1\right) * 100$

Sustituyendo los valores, se tiene:

TASA INFLACIÓN PROMEDIO

$$
\begin{aligned}
& =\left(\frac{565.01}{497.13}-1\right) * 100 \\
& =(1.13654-1) * 100 \\
& =13.65 \%
\end{aligned}
$$

Se puede notar que los valores obtenidos al calcular la tasa de inflación anual 2006 y la tasa de inflación promedio 2006, son distintos y por lo general se prestan a ser confundidos por los medios de comunicación.

- Actualización del Año Base:

Internacionalmente, se ha convenido que periódicamente se proceda con el cambio del año base del IPC, a fin de mantener y mejorar la calidad y representatividad de este indicador, motivado a los cambios que suelen experimentar las economías de los países con el transcurrir de los años. En este sentido, para 1997 (año base actual), se incorporaron los avances tecnológicos que generaron nuevos bienes y servicios (telefonía celular, servicios de internet discos compactos, entre otros), los cuales provocaron cambios en el consumo de las familias y, por tanto, en el destino y distribución de sus gastos. Ahora bien, el objetivo principal del Banco Central de Venezuela es el de combatir la inflación mediante decisiones de políticas monetarias y cambiarias dirigidas a influir sobre la demanda final de bienes y servicios de la economía (BCV, 2000).

- Valor Adquisitivo del Bolívar: (VA)

Representa los bienes y servicios que se pueden comprar con una cantidad fija de dinero (Mochón, 2001). Se calcula con la inversa del IPC multiplicada por 100.

$$
\text { VALOR ADQUISITIVO }=\left(\frac{1}{I P C}\right) * 100
$$

- Pérdida del Poder Adquisitivo del Bolívar: (PPA)

Según Mochón (2001) es la reducción del poder adquisitivo del dinero, es decir, la disminución del valor de lo que se 
puede comprar con una cantidad fija de dinero. Su fórmula para el cálculo, en este caso de estudio, es la diferencia entre el Bolívar del año base (1997) y su correspondiente valor adquisitivo, multiplicado por 100.

\section{PÉRDIDA PODER ADQUISITIVO= $(1-\text { VA })^{*} 100$}

Utilizando el IPC Promedio de cada año, a partir de 1997 (Año Base) y sustituyendo los valores en las fórmulas anteriores, se tiene lo siguiente:

\section{Tabla 1}

Poder adquisitivo y pérdida del poder adquisitivo del Bolívar Tabla del IPC (Zona Metropolitana de Caracas del BCV) Año Base 1997=100

\begin{tabular}{cccc}
\hline Año & $\begin{array}{c}\text { IPC } \\
\text { Promedio }\end{array}$ & $\begin{array}{c}\text { Valor } \\
\text { Adquisitivo }\end{array}$ & $\begin{array}{c}\text { Pérdida } \\
\text { Poder } \\
\text { Adquisitivo } \\
(\%)\end{array}$ \\
\hline 1997 & 100.00 & 1.00 & 1 \\
1998 & 135.78 & .73 & 27 \\
1999 & 167.79 & .59 & 41 \\
2000 & 194.98 & .51 & 49 \\
2001 & 219.41 & .45 & 55 \\
2002 & 268.63 & .37 & 63 \\
2003 & 352.15 & .28 & 72 \\
2004 & 428.73 & .23 & 77 \\
2005 & 497.13 & .20 & 80 \\
2006 & 565.01 & .18 & 82 \\
\hline
\end{tabular}

Fuente: Elaboración propia basada en información suministrada por el BCV.

La Tabla 1, muestra tanto el valor adquisitivo como la pérdida del poder adquisitivo promedio del Bolívar, referida a lo que le ocurrió a la moneda en los últimos nueve años anteriores al 31/12/2006. Si se quisiera interpretar el significado del último año, sería como sigue:

Cien bolívares (bs. 100.00) de 2006, tienen la misma capacidad de compra que tenían dieciocho bolívares (bs. 18.00) en 1997. Dicho de otra manera, para el año 2006 se ha perdido el $82 \%$ del poder adquisitivo de la moneda, con respecto al bolívar del año 1997.

En consecuencia, si se aplican las fórmulas anteriores, al IPC de octubre de 2007, con el que se cuenta para el momento de la elaboración del presente artículo, se obtienen los valores siguientes:

VALOR ADQUISITIVO (Oct/07)

$=\left(\frac{1}{I P C \text { Oct } / 07}\right) * 100$

VALOR ADQUISITIVO (Oct/07)

$=\left(\frac{1}{698.50971}\right) * 100$

$=0.143$

PÉRDIDA PODER ADQUISITIVO (Oct/07)

$=(1-$ VA Oct/07) 100

$=(1-0.14) 100$

$=86 \%$

Estos resultados muestran que cien bolívares (bs. 100) de octubre de 2007, tienen la capacidad de compra de ciento cuarenta y tres bolívares (bs.143.00) para 1997, es decir, se ha perdido el $86 \%$ del valor de compra de la moneda con respecto al bolívar de 1997.

Según Yanes (2006), los resultados obtenidos muestran cómo el principio del costo histórico, utilizado por siglos 
La inflación como factor distorsionante de la información financiera

De La Hoz, Betty; Uzcátegui, Sigilfredo; Borges, Jesús y Velazco, Angel

con éxito, dejó de cumplir con los propósitos de control y comparación que tenían para la época de su establecimiento. Este se apoya en las premisas del poder adquisitivo constante y de la estabilidad de la moneda, es decir, para una economía en la cual no exista el flagelo de la inflación. Es cuestionable pensar que actualmente con tasas de inflación de dos dígitos se cumpla con estos propósitos.

Adicional a lo anterior, la mejor evidencia de la inoperancia de los costos históricos para efectos de ser utilizados en la elaboración y comparación de la información financiera, se obtiene al observar una tabla de índice de precios al consumidor, emitida por el Banco Central de Venezuela, donde a partir de 1986 no se ha podido bajar de dos dígitos la inflación anual, con la excepción de 1996 donde el nivel alcanzado fue del $103.24 \%$. Esto indica que las operaciones de las empresas del país durante más de veinte años, están registradas mes a mes en Bolívares históricos de diferente valor adquisitivo o diferente poder de compra. Para Yanes (1993), esto significa que los estados financieros históricos no pueden ser comparados por estar constituidos por una suma de cifras distintas, en otras palabras, cifras con bolívares heterogéneos.

Es evidente, pues, que una aportación de capital por parte de los accionistas o una distribución de dividendos, durante el año 1997, con Bolívares de poder adquisitivo para ese año, hubiese sido equivalente a una cantidad mayor de bolívares si fuese realizada durante el año 2006. Lo que se observa en la Tabla 1, es aplicable a lo largo de la existencia de las empresas desde sus inicios, una vez que las mismas no solo reconozcan la inci- dencia de la inflación en los estados financieros, sino que además cuantifiquen y registren ese ajuste originado por el fenómeno inflacionario en el país, en los libros legales regidos por las disposiciones del Código de Comercio.

\section{Partidas monetarias y no monetarias}

El ajuste por inflación consiste básicamente en revaluar todas las cuentas de los estados financieros a moneda del mismo poder adquisitivo, por lo general moneda del final del periodo contable, así evitar agregar monedas de diferente poder adquisitivo. Para poder hacer estos ajustes se debe distinguir entre cuentas monetarias, las cuales están a expresadas en unidades de moneda del final del período $y$, cuentas no monetarias, las cuales están expresadas en distintas unidades de moneda correspondientes a las distintas fechas en que se crearon (Fernández, 2002).

Para Fernández (2002), las partidas monetarias son aquellas que no están protegidas contra los efectos de la inflación, puesto que sus valores nominales son constantes, salvo que estén sujetos a reajuste por contrato o decreto. En complemento a esta definición, la DPC10 menciona que las partidas monetarias reflejan el poder adquisitivo de la unidad monetaria a la fecha del último balance general. Algunas de ellas son: efectivo, cuentas por cobrar, cuentas por pagar, préstamos, entre otras.

En este mismo orden de ideas, Catacora (2000) indica que las partidas de naturaleza monetaria pierden valor en economías que tienen una alta tasa de in- 
flación, y como todo fenómeno económi$\mathrm{co}$, debe existir una contraparte que gana valor al transferirlas a su poseedor. Su valor histórico está representado por un monto fijo nominal que no cambia, y una parte de ese valor nominal se pierde por efectos de la inflación.

En otras palabras, las partidas monetarias son aquellas que al cierre de un periodo determinado están expresadas automáticamente a los niveles de poder adquisitivo de la moneda en ese momento y por lo tanto, con el transcurso del tiempo, pierden valor por el hecho de estar expresadas y ser representativas de moneda corriente.

Por otra parte, Fernández (2002) define partidas no monetarias como aquellas que están protegidas contra los efectos de la inflación y están expresadas normalmente en distintas unidades de moneda. Catacora (2002) complementa esta definición diciendo que son partidas que aumentan su valor en épocas de inflación sostenida y que no expresan un valor fijo en términos de la unidad utilizada como signo monetario de la economía en la cual se desarrolla una entidad.

Por otro lado, aunque la DPC-10 no define explícitamente lo que debe considerarse como partidas no monetarias, en el párrafo 15 hace referencia a ellas como sigue:

"Los activos no monetarios tienden a guardar su valor productivo aparte de las fluctuaciones en el nivel general de precios. Ejemplos son las existencias y el activo fijo. Los pasivos no monetarios se limitan a ciertos criterios diferidos las obligaciones sujetas a reajuste en función del nivel general de pre- cios. Bajo el concepto de precios constantes, su valor histórico es su costo (u otra base al originarse) dividido entre el nivel de precios en ese entonces multiplicado por el nivel de precios a la fecha. Se habla de reexpresar estas partidas, como también las monetarias a fechas anteriores".

Más adelante, la DPC-10 en su párrafo 65 agrega que las partidas no monetarias también representan aquellas cuentas de resultados que surgen de la asignación entre períodos de los activos y pasivos no monetarios. Por ejemplo: costo de venta, depreciación de activo fijo, amortización de costos prepagados y saldos de los diferidos no monetarios.

Sin duda, el primer paso para ajustar un estado financiero con cifras históricas debe ser clasificar las partidas en monetarias y no monetarias. Para ello Catacora (2002), a manera de resumen establece unas reglas básicas de clasificación:

- Todas aquellas partidas cuyo valor nominal es constante se consideran monetarias.

- Las partidas cuyo valor nominal difiere con respecto a su verdadero valor, se consideran no monetarias.

- Las partidas que se deriven de partidas no monetarias, se tratan como no monetarias.

- Las partidas del activo, deben ser analizadas una a una para determinar su clasificación en monetaria o no monetaria para efectos del ajuste.

- Por lo general, la mayoría de las partidas del pasivo son de naturaleza monetaria, una excepción son los créditos diferidos. 
La inflación como factor distorsionante de la información financiera

De La Hoz, Betty; Uzcátegui, Sigilfredo; Borges, Jesús y Velazco, Angel

- Las partidas del patrimonio por lo general son consideradas de naturaleza no monetaria.

- En el estado de resultados, las partidas no monetarias son aquellas que se derivan de partidas no monetarias del balance general, las demás son monetarias.

Las definiciones de partidas monetarias y no monetarias ya expuestas en los párrafos anteriores bajo este subtítulo, ayudan a entender que para una empresa poder determinar si los efectos de la inflación sobre cifras históricas fueron favorables o desfavorables, es necesario realizar una clasificación y análisis previo de aquellas partidas que no están protegidas contra la inflación, es decir, las monetarias. Esto con la finalidad de conocer en un momento determinado el resultado monetario por exposición a la inflación una vez actualizadas las cifras con métodos de ajustes.

Por ejemplo, para una empresa que opere bajo economías inflacionarias, es mejor tener pasivos monetarios que activos monetarios, ya que los pasivos monetarios representan un dinero que se debe a otra persona y que en el momento del pago ya ha perdido poder adquisitivo. Cuando se realiza la cancelación de la deuda se está trasladando el dinero (partida no protegida contra los efectos de la inflación) a manos de otra persona, lo que permite reflejar una ganancia monetaria. Este ejemplo deja ver que, sin una previa clasificación de las partidas en monetarias o no monetarias, sería imposible determinar si una empresa gana o pierde por efectos de inflación.

\section{Posición monetaria y sus movimientos}

Después de recalcar la importancia de clasificar las partidas que conforman los estados financieros en monetarias y no monetarias, se estudiará con detenimiento un término que es fundamental para obtener un resultado monetario del ejercicio por exposición a la inflación: La posición monetaria. La cuantificación correcta del efecto por posición monetaria está condicionada por la adecuada segregación de las partidas monetarias de las no monetarias.

Rodríguez (1999) define posición monetaria neta como la diferencia entre los activos y pasivos monetarios en un momento determinado. Redondo (1993) y Catacora (2002) comparten la misma definición que Rodríguez (1999). En concordancia con estos tres autores se puede decir entonces, que la posición monetaria va a representar un resultado (positivo o negativo), producto de los cambios en el poder adquisitivo de las partidas monetarias activas y pasivas debido a que su valor nominal es constante.

Con respecto a esto, la DPC-10 en su párrafo 40 establece que la ganancia o pérdida monetaria es la consecuencia de exponer la posición monetaria neta de una entidad a lo largo de un periodo de fluctuación del poder adquisitivo del dinero. En ese mismo párrafo se explica que la posición monetaria puede ser activa cuando los activos monetarios superan los pasivos monetarios es pasiva cuando los pasivos monetarios superan los activos monetarios. 
Catacora (2002) también menciona dos tipos de posición monetaria:

- Posición monetaria activa: Se presenta cuando los activos monetarios son mayores que los pasivos monetarios.

- Posición monetaria pasiva: Se presenta cuando los pasivos monetarios son mayores que los activos monetarios.

Rodríguez (1999), al igual que la DPC-10 y Catacora (2002), también explica dos tipos de posición monetaria sólo que les da otra denominación. Dice que puede ser positiva o negativa. Es positiva cuando los activos exceden a los pasivos es negativa cuando los activos son menores que los pasivos.

Por el contrario, Redondo (1993) habla de tres tipos de posición monetaria:

- Posición monetaria positiva o activa: Se considera positiva cuando los activos monetarios son superiores a los pasivos monetarios. Esta posición representa una pérdida para la entidad en épocas de inflación.

- Posición monetaria negativa o pasiva: Se considera negativa cuando los pasivos monetarios son superiores a los activos monetarios. Esta posición representa una ganancia para la entidad en épocas de inflación.

- Posición monetaria neutra: Se considera neutra cuando los activos monetarios tienen el mismo valor al de los pasivos monetarios.

En concordancia con la clasificación de las posiciones monetarias ya expuestas, en un periodo de inflación, toda empresa que mantenga un exceso de activos monetarios sobre pasivos monetarios, perderá poder adquisitivo, y toda empresa que mantenga un exceso de pa- sivos monetarios sobre activos monetarios, ganará poder adquisitivo, siempre que tales partidas no se encuentren sujetas a un índice de precios.

En tal sentido, el efecto por posición monetaria es producto de decisiones financieras tomadas por las empresas. Nace de la existencia de activos monetarios y pasivos monetarios que durante una época inflacionaria han disminuido su poder adquisitivo, al mismo tiempo que siguen manteniendo su valor nominal.

En el caso de los activos monetarios esto provoca una pérdida, ya que cuando la empresa los convierta en dinero o haga uso del mismo, dispondrá de una cantidad igual al valor nominal de éstos pero con un poder adquisitivo menor. Por el contrario, con los pasivos el efecto es favorable para la empresa, ya que liquidará un pasivo con cantidades de dinero de menor poder adquisitivo.

De modo que pareciera que a una entidad siempre le convendría tener una posición monetaria neta pasiva para generar ganancias en inflación, sin embargo, Catacora (2002) no comparte del todo esta aseveración. Para él no es lógico desde el punto de vista económico, ya que debe recordarse que existen algunas reglas contables y financieras que van contra esto y que ponen a evaluar la real capacidad de endeudamiento de una entidad.

\section{La ecuación fundamental y su interrelación con la actualización de la información financiera}

La Contabilidad mide los cambios básicos de los estados financieros considerando tres elementos: los activos (bienes y derechos a una fecha); los pasivos 
La inflación como factor distorsionante de la información financiera

De La Hoz, Betty; Uzcátegui, Sigilfredo; Borges, Jesús y Velazco, Angel

(deudas u obligaciones a una fecha) y el patrimonio (aportes de capital social más o menos resultado del ejercicio).

Dados estos elementos, se pueden establecer los diferentes escenarios obtenidos de la ecuación fundamental contable:

a) Activo $=$ Pasivo + Patrimonio, plantea independencia entre los recursos de la entidad y los recursos de sus dueños.

b) Patrimonio $=$ Activo - Pasivo, destaca la participación de los dueños y su importancia para el análisis del mantenimiento del capital.

c) Pasivo = Activo - Patrimonio, se utiliza para el análisis de operaciones.

Considerando el segundo escenario $($ Patrimonio $=$ Activo - Pasivo $)$, dada su importancia para el análisis del mantenimiento del capital, se tiene:

a) Patrimonio = Activo - Pasivo. Esta igualdad, al incluir los conceptos bási- cos presentes en la actualización de la información financiera, se mostraría de la manera siguiente:

b) Patrimonio = (Activo Monetarios + Activos No Monetarios) - (Pasivos Monetarios + Pasivos No Monetarios)

Ignorando los Pasivos No Monetarios, por constituir normalmente conceptos que han sido hechos con unidades monetarias cuyo valor ha venido reduciéndose, resulta evidente la necesidad de reexpresarlos en función de los factores inflacionarios aplicables. Considerando las diversas épocas en que surgieron individualmente, se tiene lo siguiente:

Fórmula General:

Patrimonio neto + Pasivos Monetarios $=$ Activos Monetarios + Activos No Monetarios

Asignando valores, se obtendría:

\begin{tabular}{|c|c|c|c|c|c|c|}
\hline Patrimonio Neto & + & $\begin{array}{c}\text { Pasivos } \\
\text { Monetarios }\end{array}$ & $=$ & $\begin{array}{c}\text { Activos } \\
\text { Monetarios }\end{array}$ & + & $\begin{array}{l}\text { Activos No } \\
\text { Monetarios }\end{array}$ \\
\hline 600 & + & 400 & $=$ & 300 & + & 700 \\
\hline \multicolumn{7}{|c|}{ a)Posición Monetaria Equilibrada } \\
\hline \multicolumn{3}{|c|}{ Pasivos Monetarios } & $=$ & \multicolumn{3}{|c|}{ Activos Monetarios } \\
\hline \multicolumn{3}{|c|}{300} & $=$ & \multicolumn{3}{|c|}{300} \\
\hline \multicolumn{7}{|c|}{ b)Posición Monetaria Activa o Larga } \\
\hline Pasiv & one & & $<$ & \multicolumn{3}{|c|}{ Activos Monetarios } \\
\hline & & & $<$ & \multicolumn{3}{|c|}{400} \\
\hline \multicolumn{7}{|c|}{ c)Posición Monetaria Pasiva o Corta } \\
\hline Pasiv & one & & $>$ & \multicolumn{3}{|c|}{ Activos Monetarios } \\
\hline & & & $>$ & \multicolumn{3}{|c|}{200} \\
\hline
\end{tabular}


Los ejemplos que se presentan a continuación, permitirán explicar lo que en realidad sucede en el balance general de las empresas, cuando cada tipo de posición monetaria posible se presenta bajo

- Posición Monetaria Equilibrada

a) Antes de la reexpresión, sin inflación:

Activos Monetarios 300

Activos No Monetarios $\quad \underline{700}$

1000

b) Después de la reexpresión, con inflación:

$\begin{array}{rr}\text { Activos Monetarios } & 300 \\ \text { Activos No Monetarios } & \underline{819} \\ (700 \times 1.1697) & 1119\end{array}$

- Posición Monetaria Activa o Larga

a) Antes de la reexpresión, sin inflación:

$\begin{array}{lr}\text { Activos Monetarios } & 400 \\ \text { Activos No Monetarios } & \frac{600}{1000}\end{array}$

b) Después de la reexpresión, con inflación:

$\begin{array}{ll}\text { Activos Monetarios } & 400 \\ \text { Activos No Monetarios } & 702\end{array}$

$(600 \times 1.1697)$

\section{2}

- Posición Monetaria Pasiva o Corta

a) Antes de la reexpresión:

$\begin{array}{lr}\text { Activos Monetarios } & 200 \\ \text { Activos No Monetarios } & \frac{800}{1000}\end{array}$

b) Después de la reexpresión:

Activos Monetarios 200

Activos No Monetarios 936

$(600 \times 1.1697)$ dos escenarios, uno sin inflación y el otro con la inflación; utilizando como supuesto la inflación con la que terminó el año 2006 correspondiente al $16,97 \%$.
300

$\underline{700}$

1000

300

$\begin{array}{lr}\text { Patrimonio Neto } & \frac{819}{1119}\end{array}$

$(700 \times 1.1697)$

Pasivos

300

Patrimonio Neto $\quad \mathbf{7 0 0}$

1000

Pasivos $\quad 300$

Patrimonio Neto 819

$(700 \times 1.1697)$

Resultado por

Posición Monetaria $\quad \leq 17>$

1102

Pasivos

300

Patrimonio Neto $\quad \underline{700}$

1000

Pasivos 300

Patrimonio Neto 819

$(700 \times 1.1697)$

Resultado por

Posición Monetaria $\quad \frac{17}{1136}$ 
La inflación como factor distorsionante de la información financiera

De La Hoz, Betty; Uzcátegui, Sigilfredo; Borges, Jesús y Velazco, Angel

De lo antes expuesto se establece que:

a) En Posiciones Monetarias equilibradas el resultado por Posición Monetaria es nulo.

b) En Posiciones Monetarias activas o largas, la insuficiencia de los Activos No Monetarios para respaldar la actualización del patrimonio neto, origina un resultado desfavorable por Posición Monetaria.

c) En Posiciones Monetarias pasivas o cortas, al actualizarse el exceso de activos no monetarios con respecto al patrimonio neto, produce un resultado favorable por Posición Monetaria.

Ahora bien, al llevar los resultados obtenidos al cuerpo del Balance General, se obtiene lo siguiente: ción, tanto a usuarios internos como externos. Sin embargo, en economías inflacionarias los estados financieros basados en costos históricos, no permiten que la información sea veraz, actual, relevante y precisa.

Todos los países, en algún momento de su historia económica, han experimentado las consecuencias que trae un proceso inflacionario sobre las cifras reflejadas en los estados financieros. Es por esta razón que se ha definido la inflación como un fenómeno económico que genera diversas consecuencias negativas, como la pérdida del poder adquisitivo de la moneda o del signo monetario representativo de una economía, que obliga a las empresas a tomar acción sobre la presentación de su información financiera.

a) En Posiciones Monetarias equilibradas:

Reexpresión del Patrimonio Neto = Reexpresión de los Activos No Monetarios

b)En Posiciones Monetarias Activas:

Reexpresión del Patrimonio Neto $\quad=\quad$ Reexpresión de los Activos No Monetarios + Resultado desfavorable por Posición Monetaria

c)En Posiciones Monetarias Pasivas:

Reexpresión del Patrimonio Neto $=$ Reexpresión de los Activos No Monetarios Resultado favorable por Posición Monetaria

\section{Conclusiones}

Los estados financieros que se derivan de la contabilidad, constituyen el medio principal para comunicar información acerca de la situación financiera de una empresa y sus resultados de opera-
Cualquier empresa ve necesariamente afectada su situación patrimonial como consecuencia de actuar en entornos inflacionarios, por lo cual todo análisis que se haga de sus cifras presentadas en los estados financieros, debe basarse en cifras actualizadas o reexpresadas 
que sean válidas y relevantes desde el punto de vista financiero, para la toma de decisiones. Para ello, se requiere de la aplicación de técnicas para conocer los niveles de inflación alcanzados por países con inflación. En Venezuela, por ejemplo, la inflación se mide a través de la variación porcentual del Índice de Precio al Consumidor (IPC) durante un periodo determinado, bien sea un mes, trimestre, semestre o año.

Con dichos IPC se puede determinar el factor de actualización de las cifras, la inflación anual, la inflación promedio, el valor adquisitivo de la moneda, la pérdida del poder adquisitivo de la moneda, entre otros conceptos. En otras palabras, la presencia de los IPC facilita el trabajo de actualización o reexpresión de estados financieros independientemente de la metodología de ajuste aplicada en cada empresa, por ser un índice basado en los precios de la canasta de bienes y servicios representativa del consumo familiar, durante un periodo determinado.

El ajuste por inflación consiste básicamente en revaluar todas las cuentas de los estados financieros a moneda del mismo poder adquisitivo, Para poder hacer estos ajustes se debe hacer una distinción clara entre cuentas monetarias y no monetarias. Las partidas monetarias son aquellas que no están protegidas contra los efectos de la inflación, puesto que sus valores nominales son constantes; por el contrario, las partidas no monetarias sí están protegidas contra los efectos de la inflación y están expresadas normalmente en distintas unidades de moneda.

Es importante saber que sin una previa clasificación de las partidas en monetarias o no monetarias, sería imposible deter- minar si una empresa gana o pierde por efectos de inflación. Para determinar el resultado monetario del ejercicio por exposición a la inflación, es necesario conocer la posición monetaria de la empresa, que se calcula restando los activos y pasivos monetarios en un momento determinado. Dicha posición va a representar un resultado positivo o negativo, producto de los cambios en el poder adquisitivo de las partidas monetarias activas y pasivas debido a que su valor nominal es constante.

Hoy en día es menor la resistencia de aceptar fácilmente el hecho de que mantener depositado dinero durante cualquier periodo de tiempo, se traduce en una pérdida del valor del mismo. Sin embargo, es difícil reconocer una ganancia resultante de la posición monetaria, puesto que se alega que ésta no se refleja en ninguna cuenta bancaria por distribuir.

En tal sentido, las posiciones monetarias pueden ser activas, pasivas o equilibradas. Toda empresa que mantenga un exceso de activos monetarios sobre pasivos monetarios, estará en presencia de una posición activa y perderá poder adquisitivo. Si el exceso es de pasivos monetarios sobre activos monetarios, la posición es pasiva y ganará poder adquisitivo. Cuando los activos monetarios igualan a los pasivos monetarios la posición es equilibrada generándose una situación en la que no se gana ni se pierde.

Esto permite llegar a la conclusión de que en posiciones monetarias activas, la insuficiencia de los activos no monetarios origina un resultado desfavorable por posición monetaria. Asimismo, en posiciones monetarias pasivas o cortas, al actualizarse el exceso de activos no monetarios con respecto al capital contable, se 
La inflación como factor distorsionante de la información financiera

De La Hoz, Betty; Uzcátegui, Sigilfredo; Borges, Jesús y Velazco, Angel

produce un resultado favorable por posición monetaria y en posiciones monetarias equilibradas el resultado por posición monetaria es nulo.

En consecuencia, todo aquel que administre una empresa, invierta, quiera invertir en ella o se le exija el pago de impuestos derivados de los beneficios de la misma, debe saber medir el impacto de cómo le afecta la inflación; estar consciente si los cambios en el patrimonio, provienen de los resultados de la relación precio-producto o precio-servicio ofrecidos o de las variaciones en la unidad de medida y registro, que en el caso venezolano es el Bolívar. En otras palabras, la ganancia o pérdida neta de una empresa que se desenvuelve en un entorno inflacionario como el de Venezuela, está formada por dos componentes distintos: la comercialización de los productos y/o servicios ofrecidos y el tratamiento financiero que la misma le ha dado a las partidas monetarias y no monetarias durante el ejercicio, donde la inflación de por si es el ingrediente esencial.

\section{Referencias Bibliográficas}

Banco Central de Venezuela (2000). Índice de precios al consumidor, año base 1997. El termómetro de la inflación. www.bcv.org.ve. Consulta: Agosto 21 de 2007.

Banco Central de Venezuela (2000). IPC: seguimiento confiable de la inflación. Boletín económico informativo. Marzo - Abril 2006. Año 11.

Catacora Fernando (2000). Reexpresión de Estados Financieros. McGraw-Hill Interamericana de Venezuela, S.A. Colombia.

Centro de Estudios Latinoamericanos (CESLA) (2007). Tendencias latinoameri- canas. www.cesla.com. Consulta: Agosto 21 de 2007.

Dornbusch Rudiger; Fischer Stanley; Startz Richard (2002). Macroeconomía. McGraw-Hill Interamericana de Venezuela, S.A. Octava edición. España.

Federación de Colegios de Contadores Públicos de Venezuela. Declaración de Principios de Contabilidad No. 10 (DPC-10). (1991), reemplazada en su totalidad por la normativa revisada e integrada el 06/12/2000.

Fernández Jesús (2002). Reexpresión de Estados Financieros en Venezuela. Enfoque práctico. Venezuela.

Mochón Francisco (2001). Economía teoría y política. Cuarta edición. McGraw-Hill.

República Bolivariana de Venezuela (2006). Banco Central de Venezuela. Informes Mensuales de IPC. Www.bcv. org.ve. Consulta: Agosto 21 de 2007.

Redondo Angel (1993). Curso práctico de contabilidad general superior. Tomo II. Tercera edición. Venezuela

Rodríguez Luis Antonio (1999). La influencia de la inflación en la contabilidad. Trabajo final nivelatorio de la contabilidad. (http://www.geocities.com). Consulta: Septiembre 14 de 2007, hora: 6:00 p.m.

Vera Leonardo (1996). Contribuciones al análisis de la inflación. Anotaciones para el caso venezolano. Ediciones EVE. Venezuela.

Vives Antonio (2003). Evaluación financiera de empresas. El impacto de la devaluación la inflación. Sexta reimpresión. Editorial Trillas. México.

Yanes José Alberto (2006). Guía de ajuste por inflación financieros y fiscales. Clemente editores, C.A. Valencia, Venezuela.

Yanes José Alberto (1993). Manual de ajuste por inflación. Clemente editores, C.A. Valencia, Venezuela. 